\title{
Development and Achievement of the T-50 Flight Control's Consolidated OFP
}

\author{
Soon Ryong Jang, In Je Cho and Byung Moon Hwang \\ Flight Control Team, Korea Aerospace Industries, LTD., Sacheon, Korea
}

\begin{abstract}
The OFP (operational flight program) of the digital fly-by-wire FCS (flight control system) system is safety-critical item and has to be designed and verified to assure the safety of flight based on tightly coupled process. T-50 FCS OFP interacts with triplex redundant FCS system and other major subsystem including avionics. So far, the multi-version of FCS OFP has been developed and serviced per each T-50 derivatives' requirements. The consolidation of OFP was demanded to save development cost and schedule, by concurrent release to service all T-50 derivatives when general improvement is requested. Through successful developing the Plug \& Play type OFP, it is possible to maintain the single configuration of FCS OFP as well as compatibility with dissimilar avionics of each T-50's derivatives. In this paper, we present the development process, results and achievement of the consolidated FCS OFP.
\end{abstract}

Key words: FCS, OFP, Plug \& Play type.

\section{Introduction}

The flight control system and OFP of a highly maneuverable fighter class aircraft is critical item which directly impacts on aircraft safety. Flight control software controls or monitors hardware whose reliability or performance directly impacts on the PLOC (probability-of-loss of control), survivability, aircraft performance (stability \& handling qualities) and crew safety (structural, loads, and human limitations) [1].

T-50 Flight control system design encompasses the interface with major subsystem to acquire the accurate control and integrity. The major interacting subsystem consists of hydraulic, electrical, avionics, engine, landing gear, cockpit system, etc., and also support equipment. The T-50 was originally developed for LIFT (lead-in fighter like trainer), modified to TA-50 supporting CAS (close air combat) mission, and finally upgraded to the FA-50 for air to air combat mission and electric warfare. Other variants are T-50B for ROKAF acrobatic team, T-50i for

Corresponding author: Soon Ryong Jang, flight control system design lead \& senior research engineer, main research field : flight control system, safety critical software mechanization. E-mail: s.r.jang@koreaaero.com.
Indonesian AF (air force), FA-50PH based on FA-50 for Philippine AF. To satisfy the customer requirement of these $\mathrm{T}-50$ variants, aircraft subsystem including flight control system need to improve. Most of changes so far are generally required in the avionics system.

In this paper, development and achievement of the plug \& play type consolidated flight control OFP are addressed. Through successful developing the Plug \& Play type OFP, it is possible to maintain the single configuration of FCS OFP as well as compatibility with dissimilar avionics of each T-50's derivatives. In this paper, we present the development process, results and achievement of the consolidated FCS OFP as a best practice.

\section{Consolidated OFP's Needs}

The flight control system is responsible for controlling flight, attitude and safety. The avionics system is responsible for communication/navigation/weapon/display, inter-commuting the aircraft information (air data, motion and states) for weapon delivery, navigation and the navigation, pilot display interface for fine control and autopilot mode via MIL-STD-1553B 
MUX Bus. When the IMDC (integrated mission computer) fails, the FLCC (flight control computer), a highly reliable, safe and high computing performance device, will provide backup bus control capability for maintaining transaction on the Avionics 1553 MUX Bus. Through this safety feature, the engine, pilot primary display, navigation, flight data record, and weapon delivery mission are able to continue under mission computer loss.

As a result of that, when each variant has expanded or improved the avionics system design, the backup bus control design becomes dissimilar in each flight control OFP and this impacts the interface. From this background, the consolidated flight control OFP was proposed to integrate all variants' backup bus control requirement but maintain the single or common version for assuring flight safety critical feature as well as saving maintenance cost and development effort in the life cycle. From the results of a tradeoff study, the addition of 4 independent aircraft signals were selected to identify each variant or block of the T-50 series as shown in Fig. 1 [2]. The 4 independent signals have triplex redundancy and are used to switch the proper configuration database per each variant.

\section{Development and Achievement of the}

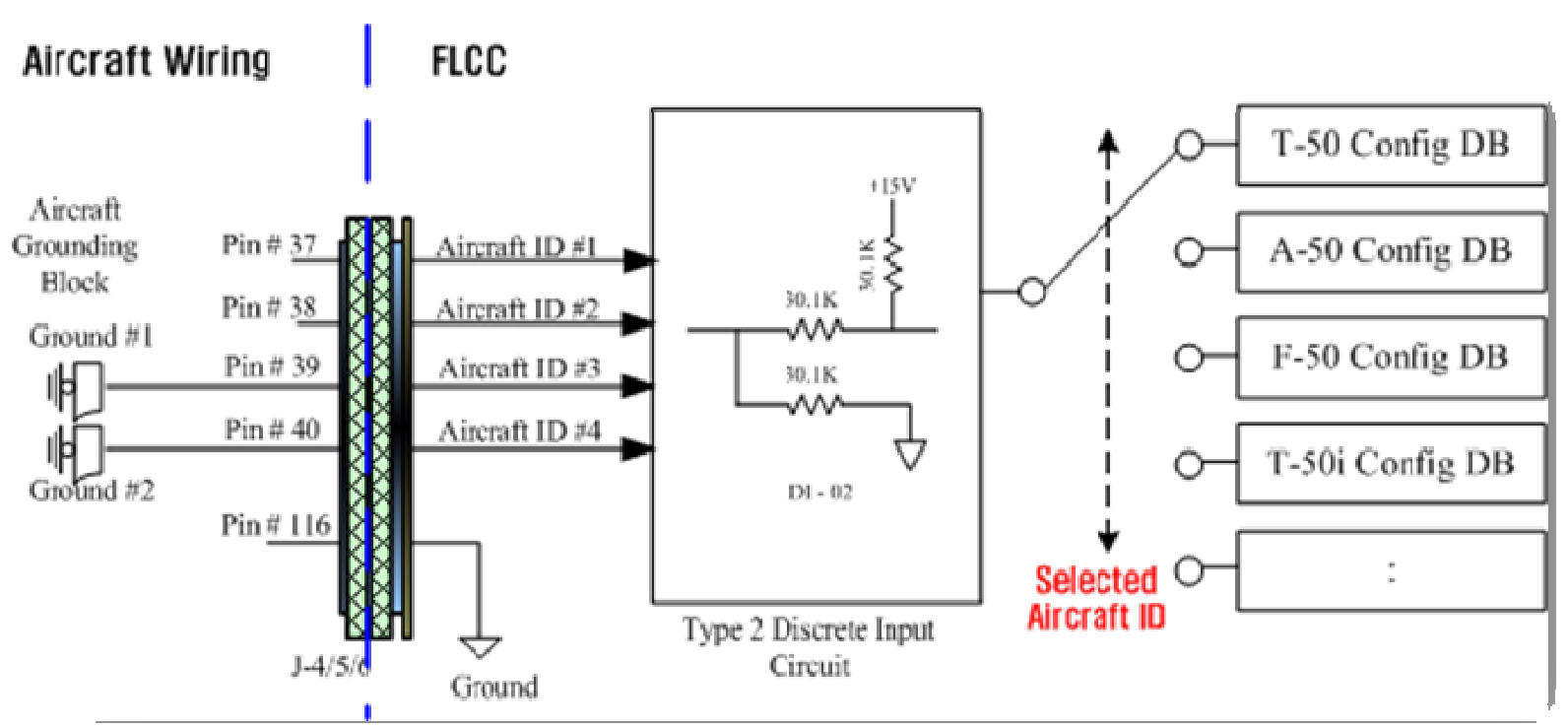

\section{Consolidate Flight Control OFP}

The below Fig. 2 is to show the conceptual structure of plug \& play type, consolidated flight control OFP which will compatible with multiple dissimilar aircraft and subsystems.

The design feature for each functional module is summarized in the following.

Aircraft wiring module is to implement the identification code using independent, redundant aircraft wirings and input to the flight control computer and select the aircraft identification configuration. Power up CSC (computer software component) Module will determine the proper aircraft identification and initialize all internal parameter during boot up of the flight control computer. The AVS 1553 CSC module and provisional CSC will determine and switch the subsystem interface parameter with the appropriating parameter database per preselected aircraft identification at power up stage and also encompasses the other variant-dependent potential design features.

Signal management CSC module will perform the signal fault detection monitor and select the aircraft identification by voting rule after receiving the aircraft wiring input signal as discrete type. Built-in-test CSC

Fig. 1 Aircraft wiring design for the aircraft identification. 


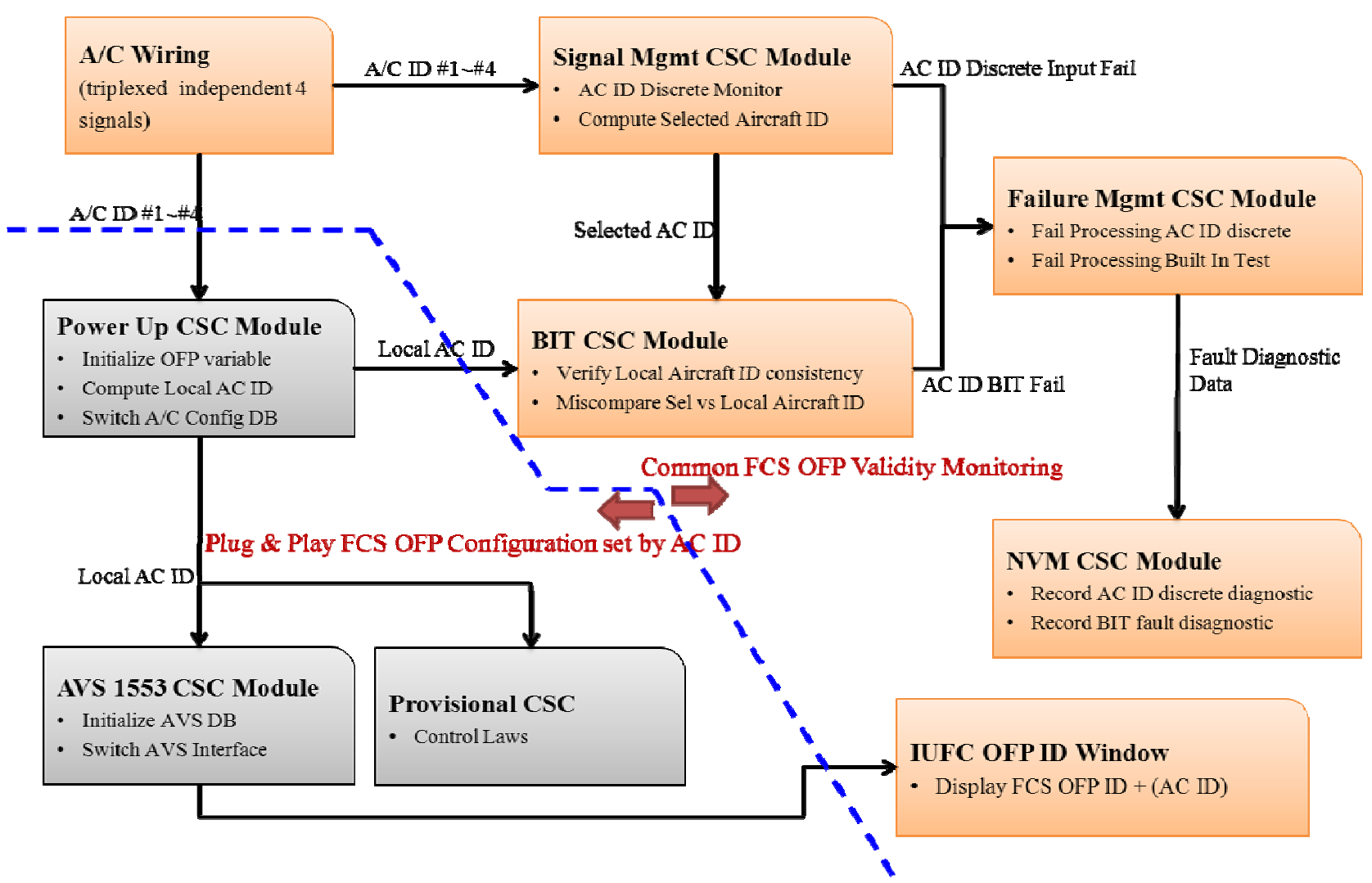

Fig. 2 Conceptual structure of the consolidated OFP.

Module is generally initiated by pilot at preflight checkout stage for the flight mission. It will validate the correctness of initialized and configured aircraft identification and its database, and also checks whether the each module processing of consolidated OFP is ready for flight or not.

Failure Management CSC Module will process the failure detection reporting and diagnostic information resulting from Signal Management CSC and BIT CSC. It will analyze the fault impact at the system level, isolate the fault to prevent the fault propagation and report the fault isolation results to the pilot or operator with caution lamp and fault codes using cockpit advisory and displays. Then pilot or operator will take corrective action in accordance with fault reporting.

NVM CSC Module will record all diagnostic datum related to the fault of aircraft identification or improper operation from misconfiguration and all referenced sources for maintaining the aircraft identification value under any transient or emergency situation. This data will used to troubleshoot and investigate the root cause of the systematic fault. IUFC (integrated up front control) will display the flight control OFP ID which contains the T-50 variant's aircraft identity code at the last text. From this positive design feature, pilot or operator can inspect the FCS OFP working status by checking aircraft identity code prior to every flight mission.

The flight and safety critical flight control OFP development thoroughly follow the proven SOF (safety-of-flight certificate) process as shown in Fig. 3. The validation and verification activities had been completed without any hazardous SPAR (system/software product anomaly report) for the consolidated flight control OFP.

Per the above SOF certification process, the software level unit test was completely passed and the hardware/software integration test was successfully completed with real time and real platform environment. For further design validation purpose, SFMEA (software 


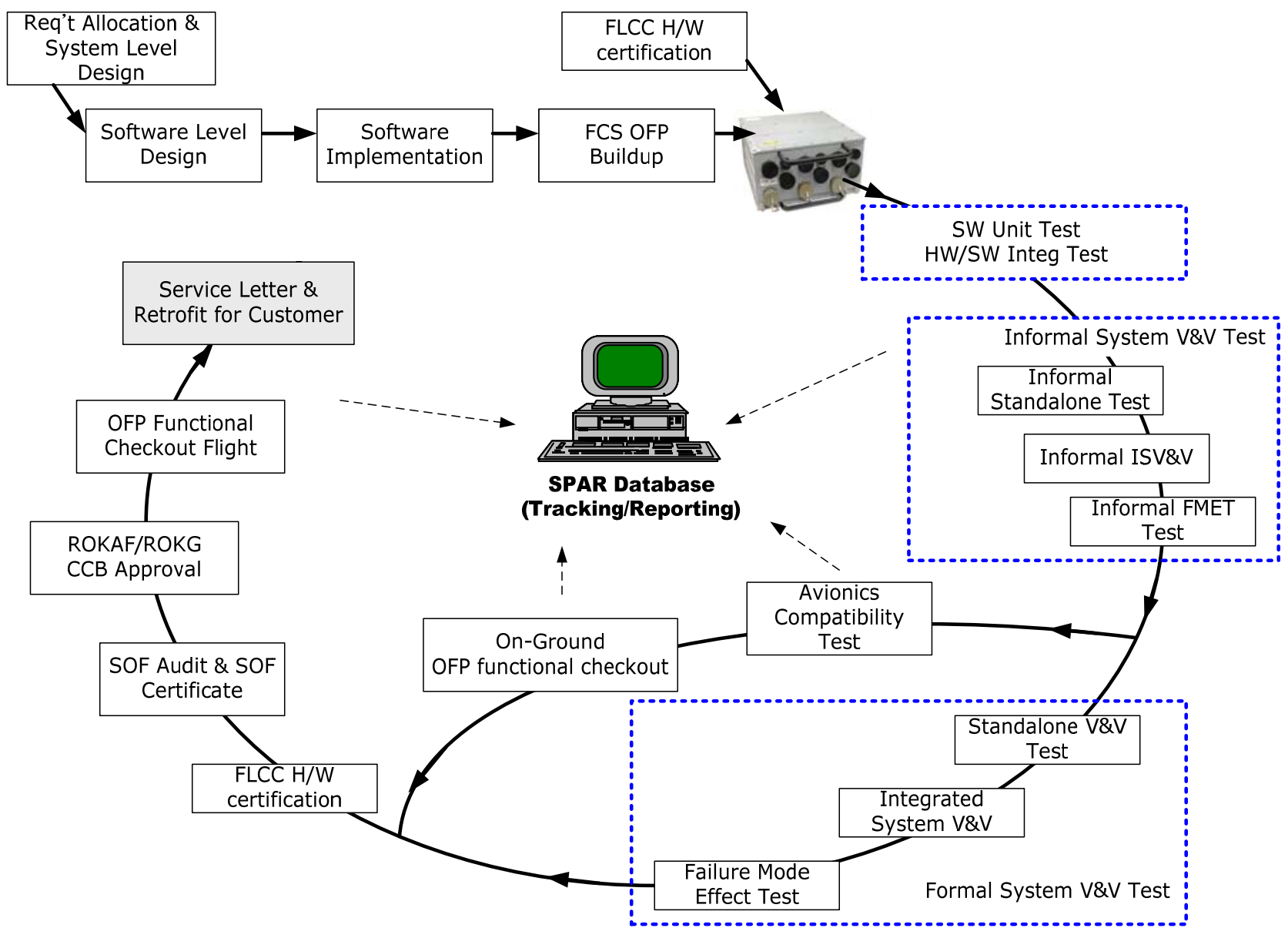

Fig. 3 Safety of flight certification flow for flight critical FCS OFP.

failure mode effect analysis) was performed to identify the potential hazard of the consolidated OFP and incorporate those hazard cases into design validation test procedure [3].

Since each variant OFP configuration matching with aircraft identification should be verified per flight critical FCS OFP development process, conceptually total demands of test schedule and effort are multiplied by the number of variants. The $\mathrm{V} \& \mathrm{~V}$ (validation and verification) test for flight control OFP is composed of three different test categories. The first test is SAVV (stand-alone test), FCS (unit under test) in an open loop environment to verify the software functional requirements. The second test is ISVV (integrated system test), hardware-in-the-loop integration simulation used to evaluate the flight-worthiness, handling qualities of the flight control OFP and verify the control laws design change. The last test is FMET (failure modes \& effects test) which demonstrates the flight controls' robustness in the presence of failures.

To mitigate this development's cost and schedule risk, a trade off study was performed to optimize the $\mathrm{V} \& \mathrm{~V}$ test case and schedule plan. According to the study results, the V\&V test cases were categorized into four different classes and finally $66 \%$ of total V\&V test cases were saved as shown in Table 1.

Category 1-Affective: Test cases were impacted by each variant configuration;

Category 2-Common: Common test cases not included in Category 1 and independent of variant configuration;

Category 3-Essential: Mandatory test case regardless of change in the FCS OFP; 
Table 1 Comparison of $\mathrm{V} \& \mathrm{~V}$ test cases.

\begin{tabular}{llll}
\hline Test category & Savv & Isvv & Tmet \\
\hline Affected test cases & 30 & 52 & 34 \\
Common test cases & 369 & 0 & 68 \\
Essential test cases & 96 & 47 & 0 \\
Special test cases & 0 & 319 & 4 \\
Target AC IDs & 2 & 2 & 2 \\
No. of pilot & 1 & 2 & 1 \\
Single OFP total cases & 495 & 94 & 106 \\
Consolidate OFP total cases & 621 & 146 & 144 \\
Test increased rate & $126 \%$ & & \\
\hline
\end{tabular}

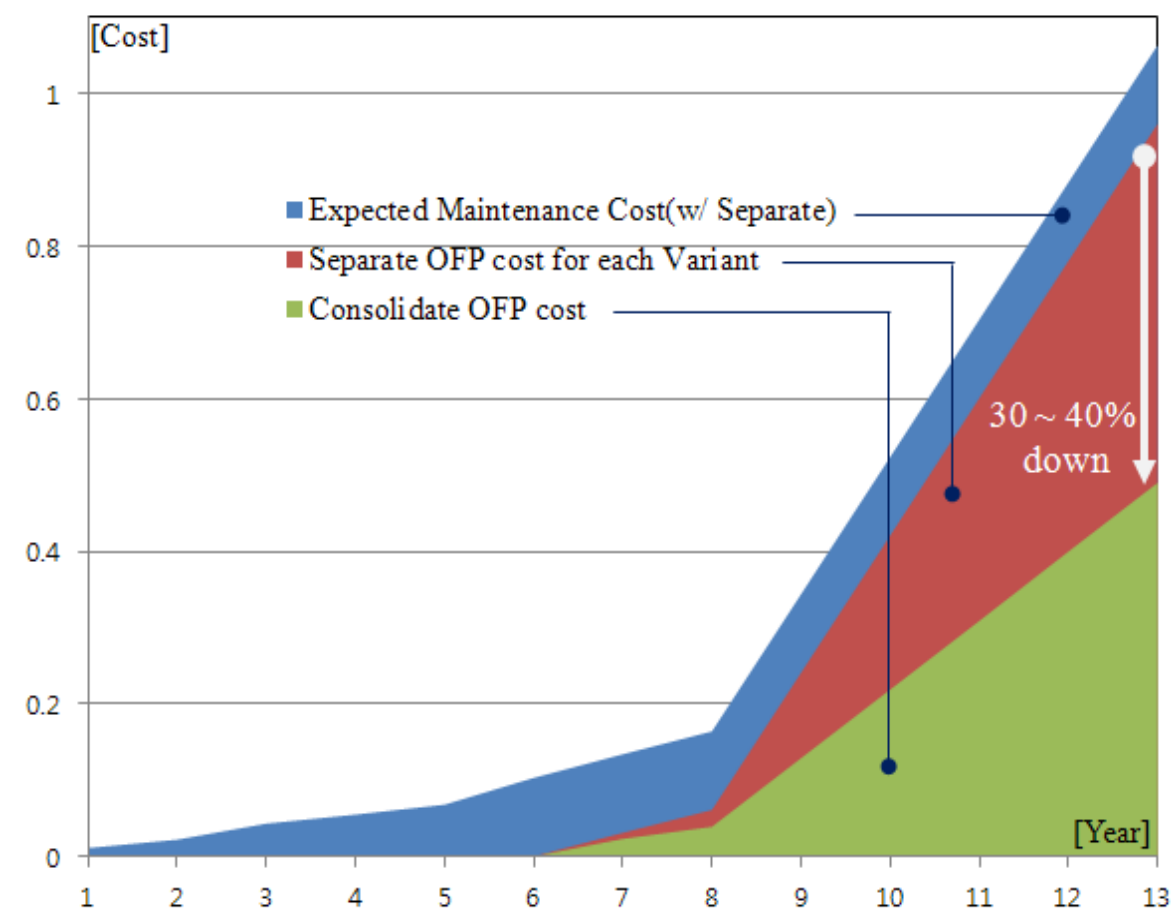

Fig. 4 Estimated life-cycle maintenance cost benefits

Category 4-Special: Test cases for verify the design change on this version, temporary check;

From the optimization of development and verification activities for developing the consolidated FCS OFP, it is possible that not only multiple and dissimilar customer requirements can be accepted but also development schedule and effort can be dramatically saved. From the statistic records, the maintenance cost for the FCS OFP in the life cycle was estimated as shown in Fig. 4.

\section{Conclusion}

The flight control OFP was successfully developed which is plug \& play type and compatible with dissimilar multiple requirement. To assure the safety and reliability of flight critical FCS OFP, the proven SOF Certification process was applied to the life cycle of FCS OFP development. Through the efforts and results of consolidated FCS OFP and process, it is possible that the development effort, schedule and maintenance cost could be saving up to approximately 30\%-40\% down versus those from existing FCS OFP way. When ROKAF (Rep. of Korea Air Force) and international customer request design improvement or new requirement in the next, single and consolidate FCS OFP can cover all dissimilar requirements of each 
variant simultaneously at the same time.

\section{References}

[1] Jang, S. R. 2012. "Digital Flight Control Software Verification Test Technology.” DAPA Weapon System Software Conference, Seoul, Korea.
[2] Jang, S. R., and Seo, S. J. 2013. "Development of the Plug \& Play Type Flight Control OFP as per Aircraft Derivatives.” KSAS Conference, Yong-pyung, Korea.

[3] Cho, S. H., and Jang, S. R. 2014. "Design Safety Advancement of the Common Flight Control OFP through Enhanced SFMEA Process Modeling.” SASE Autumn Conference, Mu-ju, Korea. 